\title{
NOUVELLE
}

\section{Contrôle du métabolisme énergétique par les peptides natriurétiques}

Cédric Moro ${ }^{1,2}$

\author{
${ }^{1}$ Inserm UMR1048, Institut des maladies métaboliques \\ et cardiovasculaires, CHU Rangueil, 1, avenue Jean Poulhès, \\ 31432 Toulouse Cedex 4, France; \\ ${ }^{2}$ Université Paul Sabatier, 118, route de Narbonne, \\ 31062 Toulouse Cedex 9, France. \\ cedric.moro@inserm.fr
}

\section{Peptides natriurétiques, obésité et diabète de type 2}

Les peptides natriurétiques (PN) sont des hormones cardiaques connues, depuis les années 1980, pour réguler la balance hydrosodée et la volémie chez les mammifères. Cette famille regroupe plusieurs membres, dont l'atrial natriuretic peptide - NP (ANP) et le brain natriuretic peptide (BNP), sécrétés respectivement par l'oreillette droite et les ventricules en réponse à une augmentation de la pression veineuse centrale et de la volémie. Les peptides natriurétiques ciblent un récepteur biologiquement actif de type A (NPRA) couplé à une activité guanylyl cyclase intrinsèque qui augmente la production intracellulaire de guanosine monophosphate cyclique (GMPc) [1]. C'est en 2000 que des travaux de notre laboratoire ont mis en évidence un rôle métabolique des peptides natriurétiques jusqu'alors insoupçonné [2]. II apparaît de plus en plus évident que ces peptides exercent, hors de la sphère cardiovasculaire, de nombreux effets biologiques dans différents tissus cibles, incluant le tissu adipeux, le muscle squelettique, le foie et le pancréas [3]. Plusieurs études épidémiologiques ont mis en évidence une association entre les niveaux circulants de peptides natriurétiques, l'obésité et le diabète de type 2 [4]. Les niveaux circulants de peptides natriurétiques diminuent graduellement avec l'augmentation de l'indice de masse corporelle et prédisent le risque de diabète de type 2 [5]. Plusieurs polymorphismes $\mathrm{du} B N P$, du récepteur biologiquement actif NPRA et du récepteur de clairance NPRC des peptides natriurétiques sont associés à l'obésité abdominale, à I'hypertension artérielle et au diabète de type 2.

\section{Régulation de la lipolyse et de la thermogenèse}

Nos travaux ont mis en évidence un effet lipolytique puissant des peptides natriurétiques sur des adipocytes humains isolés. Le mécanisme implique une activation du récepteur NPRA, une augmentation des taux intracellulaires de GMPc et une activation de l'une des enzymes limitantes de la lipolyse, la lipase hormonosensible [2]. Une injection d'ANP in situ dans le tissu adipeux sous-cutané abdominal, par microdialyse ou par voie intraveineuse, active la lipolyse et la mobilisation d'acides gras. Nous avons également montré que l'ANP, perfusé à des doses mimant les variations physiologiques, stimule la mobilisation et l'oxydation des lipides chez des volontaires sains, aussi bien à jeun qu'en période postprandiale [6]. Nous avons enfin pu établir un lien entre l'augmentation des niveaux circulants de peptides natriurétiques observée au cours d'un exercice aigu et la mobilisation des lipides [7].

Des travaux récents de l'équipe de Sheila Collins démontrent que les peptides natriurétiques stimulent le métabolisme oxydatif et induisent l'expression de la protéine découplante 1 (UCPI) dans des adipocytes humains de type hMADS (human multipotent adipose derived stem) [8]. Les auteurs ont observé une «brunisation » des dépôts adipeux blancs ${ }^{1}$ associée à une diminution de leur masse chez des souris dont le NPRC a été génétiquement inactivé $\left(N P R C^{-/-}\right)$ $[11,12]$. Ces résultats suggèrent que les peptides natriurétiques pourraient stimuler la thermogenèse. Ainsi, les niveaux circulants de BNP et l'expression du récepteur NPRA augmentent dans les tissus adipeux blancs et bruns lors d'une exposition aiguë au froid. Ces résultats pourraient avoir une pertinence physiologique chez l'homme considérant la présence de tissu adipeux brun fonctionnel.

\section{Régulation de la balance énergétique}

Des expériences menées sur des modèles animaux suggèrent que les peptides natriurétiques et la signalisation GMPc peuvent stimuler le métabolisme énergétique et protéger des souris soumises à un régime hyperlipidique de la prise excessive de masse grasse et de ses effets délétères sur la tolérance au glucose. Ainsi, la prise de poids chez des souris transgéniques ayant un niveau circulant élevé de BNP (BNP-Tg) et nourries avec un régime enrichi en lipides est atténuée par rapport à celle de souris

\footnotetext{
${ }^{1}$ « Les tissus adipeux blanc et brun diffèrent par leurs caractéristiques et leurs localisations anatomiques distinctes. Chez l'homme, le tissu adipeux brun est présent chez le fœtus et le nouveau-né et régresse ensuite rapidement. Des arguments récents indirects suggèrent cependant la persistance de tissu adipeux brun chez l'adulte. Les adipocytes blancs mettent en réserve l'excès d'énergie sous forme de triglycérides et, en situation de demande énergétique, libèrent les acides gras. Les adipocytes bruns dissipent l'énergie sous forme de chaleur. Pour assurer cette fonction particulière, les adipocytes bruns expriment dans leurs mitochondries la protéine découplante ou UCP-1 (uncoupling protein 1). De nombreuses approches réalisées chez différents modèles murins ont mis en évidence un effet anti-obésité de la stimulation du développement du tissu adipeux brun. Ainsi il est tentant de spéculer que des approches permettant de stimuler et/ou de réactiver la fonction «brune » pourraient présenter un intérêt
} thérapeutique pour limiter l'obésité » (tiré de [11]). 
contrôles [9]. Ceci pourrait s'expliquer notamment par une augmentation de la consommation d'oxygène et de l'oxydation lipidique au niveau de l'organisme entier. De plus, des souris transgéniques surexprimant globalement la protéine kinase GMPc-dépendante cGK-I sont plus minces que les souris sauvages ayant un régime standard et résistent à la prise de poids induite chez les souris sauvages par un régime gras. À l'inverse, des souris partiellement invalidées pour le récepteur biologiquement actif NPRA (NPRA $\left.{ }^{+/-}\right)$ deviennent plus susceptibles à l'obésité et moins tolérantes au glucose [9]. Chez ces souris, la prise de poids et la glycémie sont augmentées lors d'un régime hyperlipidique. Les effets protecteurs des peptides natriurétiques contre l'obésité et ses complications métaboliques pourraient être liés à une amélioration de l'utilisation des lipides et du métabolisme oxydatif dans le muscle squelettique.

\section{Régulation du métabolisme oxydatif musculaire}

Nous avons récemment observé une augmentation de l'expression du NPRA musculaire conjointement à une amélioration globale de la capacité oxydative musculaire chez des sujets obèses après huit semaines d'entraînement en endurance [10]. Nous avons ensuite montré qu'une activation de la voie des peptides natriurétiques/GMPc dans des cultures primaires de myotubes humains pouvait induire l'expression du peroxisome proliferator activated-receptor- $\gamma$ (PPAR) coactivator- $1 \alpha(\mathrm{PGCl} \alpha)$, un coactivateur transcriptionnel majeur de la biogenèse mitochondriale [13]. Le mécanisme semble impliquer une régulation transcriptionnelle de PGCl $\alpha$ par le GMPc, tandis que l'expression de PPAR $\delta$, un facteur de transcription régulant le métabolisme oxydatif, ne semble pas affectée. L'induction de PGCl $\alpha$ par les peptides natriurétiques se traduit par une activation de l'expression des gènes intervenant dans la phosphorylation oxydative. Plus intéressant, nous observons une induction de l'expression de deux protéines impliquées dans la conductance aux protons, l'adénine nucléotide translocase 1 (ANTl) et la protéine découplante 3 (UCP3), ainsi que des transporteurs des

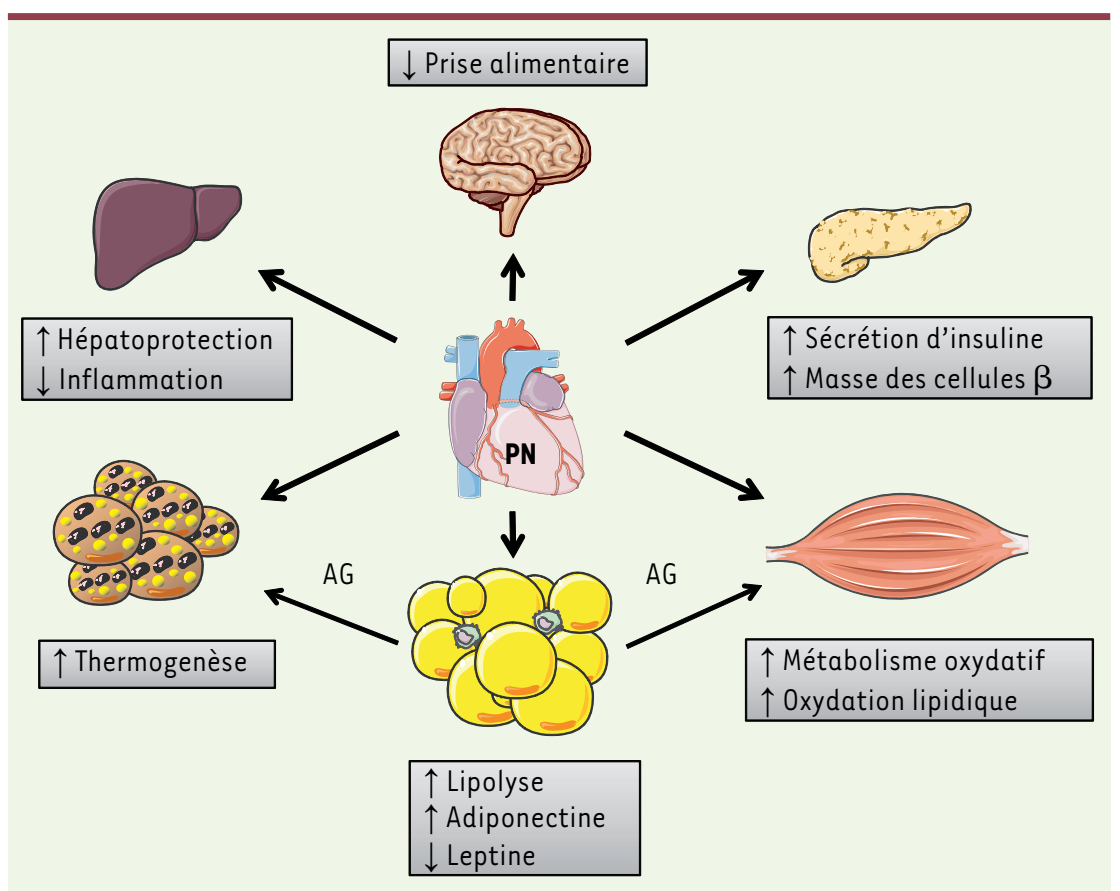

Figure 1. Schéma récapitulatif des principaux effets physiologiques des peptides natriurétiques dans le contrôle du métabolisme énergétique. AG : acides gras ; PN : peptides natriurétiques.

acides gras, fatty acid binding protein-3 (FABP3) et CD36. Ces évènements moléculaires se traduisent par une augmentation de la respiration mitochondriale maximale et découplée, et de l'oxydation des lipides [10]. Fait marquant, les peptides natriurétiques miment in vitro les effets de l'entraînement en endurance in vivo, qui s'accompagnent chez I'homme d'une augmentation du métabolisme de repos indépendamment de la masse maigre et de la consommation maximale d'oxygène.

\section{Conclusion}

Ces travaux démontrent un rôle physiologique majeur des peptides natriurétiques dans la régulation du métabolisme énergétique. Ils soulignent également un dialogue endocrine insolite entre le cœur, les tissus adipeux et le muscle squelettique coordonné par ces peptides (Figure 1). Le cœur pourrait ainsi ajuster la disponibilité en acides gras à sa propre demande ainsi qu'à celle du muscle squelettique en activité au cours de l'exercice physique. Ce système permettrait également de coupler les adaptations physiologiques cardiaques à celles du muscle squelettique en réponse à l'exercice chronique. II reste maintenant à évaluer le potentiel thérapeutique des peptides natriurétiques dans des études précliniques chez le rongeur. $\diamond$ Control of energy metabolism by natriuretic peptides

\section{LIENS D'INTÉRÊT}

L'auteur déclare n'avoir aucun lien d'intérêt concernant les données publiées dans cet article.

\section{RÉFÉRENCES}

1. Potter LR, Abbey-Hosch S, Dickey DM. Natriuretic peptides, their receptors, and cyclic guanosine monophosphate-dependent signaling functions. Endocr Rev $2006 ; 27: 47-72$.

2. Lafontan M, Moro C, Berlan M, et al. Control of lipolysis by natriuretic peptides and cyclic GMP. Trends Endocrinol Metab 2008 ; 19 : 130-7.

3. Moro C, Lafontan M. Natriuretic peptide and CGMP signaling control of energy homeostasis. Am J Physiol Heart Circ Phys 2013 (sous presse)

4. Wang TJ, Larson MG, Keyes MJ, et al. Association of plasma natriuretic peptide levels with metabolic risk factors in ambulatory individuals. Circulation 2007 ; $115: 1345-53$. 
5. Magnusson M, Jujic A, Hedblad B, et al. Low plasma level of atrial natriuretic peptide predicts development of diabetes: the prospective Malmo diet and cancer study. J Clin Endocrinol Metab 2012 ; 97 : 638-45.

6. Birkenfeld AL, Budziarek $P$, Boschmann M, et al. Atrial natriuretic peptide induces postprandial lipid oxidation in humans. Diabetes $2008 ; 57: 3199-204$

7. Moro C, Crampes F, Sengenes C, et al. Atrial natriuretic peptide contributes to physiological control of lipid mobilization in humans. FASEB J $2004 ; 18$ : 908-10.
8. Bordicchia M, Liu D, Amri EZ, et al. Cardiac natriuretic peptides act via p38 MAPK to induce the brown fat thermogenic program in mouse and human adipocytes. J Clin Invest 2012 ; 122 : 1022-36.

9. Miyashita K, Itoh H, Tsujimoto H, et al. Natriuretic peptides/cGMP/cGMP-dependent protein kinase cascades promote muscle mitochondrial biogenesis and prevent obesity. Diabetes $2009 ; 58: 2880-92$.

10. Engeli S, Birkenfeld AL, Badin PM, et al. Natriuretic peptides enhance the oxidative capacity of human skeletal muscle. J Clin Invest 2012 ; 122 : 4675-9.

\section{NOUVELL}

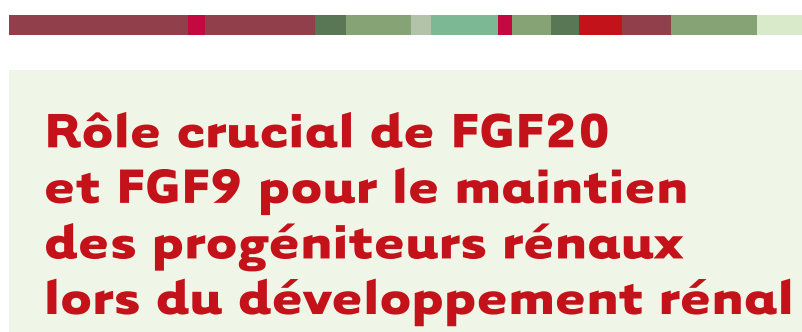

Cécile Jeanpierre

\section{Le développement rénal}

Lors du développement rénal chez les mammifères, trois ébauches se succèdent de la partie céphalique à la partie caudale du mésoderme intermédiaire de l'embryon: le pronéphros, le mésonéphros et le métanéphros. La différenciation du rein définitif ou métanéphros commence à la $5^{\mathrm{e}}$ semaine de gestation chez l'homme ( $\varepsilon 10,5$ chez la souris), et se poursuit jusqu'à la $36^{\mathrm{e}}$ semaine $\left(\approx 5^{\mathrm{e}}\right.$ jour après la naissance chez la souris). Elle est contrôlée par une induction réciproque entre une structure épithéliale dérivée du canal de Wolff, le bourgeon urétéral, et des cellules mésenchymateuses qui migrent vers l'extrémité du bourgeon urétéral pour former le mésenchyme métanéphrique condensé. Agissant de manière itérative, cette induction va permettre, d'une part, la ramification (branching) du bourgeon urétéral afin de former les canaux collecteurs et, d'autre part, la différenciation du mésenchyme métanéphrique condensé en épithélium afin de constituer les néphrons. Plusieurs stades morphologiques se succèdent (vésicule rénale, corps en virgule, corps en S) avant la formation des néphrons matures connectés aux canaux collecteurs. Le nombre de néphrons, environ un million par rein chez l'homme, est directement lié au nombre de ramifications du bourgeon urétéral. Fixé à la naissance, il est cependant d'une grande variabilité et dépend de multiples facteurs, dont l'âge gestationnel, l'état nutritionnel maternel, et également de facteurs génétiques.

\section{Gènes et voies de signalisation \\ impliqués}

De nombreux travaux réalisés grâce à des modèles animaux (souris, poisson zèbre), ex vivo (culture organotypique de métanéphros) et cellulaires, ont permis de caractériser l'implication d'un grand nombre de gènes dans le contrôle du développement rénal. Ces gènes codent notamment pour des facteurs de transcription (Wt1, Pax2, Eyal, Six1, Six2, Sall1, Hnfl $\beta$ ), pour des récepteurs et des ligands (Ret, Gdnf, Wnt9b, Wnt1l, Wnt4, $B m p 4$ ), et pour des protéines d'adhésion (cadhérines, intégrines, protéoglycanes). Une voie cruciale pour la ramification du bourgeon urétéral est la voie Ret/Gdnf [1]. De nombreux facteurs sont impliqués dans la régulation de l'expression du récepteur à activité tyrosine kinase Ret et de son ligand Gdnf (glial cell derived neurotrophic factor), permettant le position-
11. Bouloumié $A$, Sengenès $C$, Galitzky J. Les progéniteurs adipeux blancs et bruns. Med Sci (Paris) 2009 ; 25 : 123-5.

12. Nibbelink M, Arnaud E, Pénicaud L, Casteilla L. La protéine découplante du tissu adipeux brun (UCP1) : la fin des dogmes. Med Sci (Paris) $2002 ; 18$ $780-3$.

13. Tiraby C, Langin D. PGC- $1 \alpha$, un co-activateur transcriptionnel impliqué dans le métabolisme. Med Sci (Paris) $2005 ; 21: 49-54$.
Inserm U983, Institut Imagine, Université Paris Descartes, Sorbonne Paris Cité, hôpital Necker-Enfants malades, 149, rue de Sèvres, 75743 Paris Cedex 15, France. cecile.jeanpierre@inserm.fr

nement d'un bourgeon urétéral unique au départ, puis sa croissance et sa ramification (Figure 1) [2].

La différenciation épithéliale des néphrons se fait à partir d'une population de cellules progénitrices situées dans le mésenchyme métanéphrique condensé et caractérisées par l'expression des marqueurs Six2 (Six homeobox 2) et Citedl (CREB-binding protein/p300-interacting transactivator with Asp/Glu-rich C-terminal domain 1) [2] (Figure 1). Les produits d'autres gènes, tels que Wtl (Wilms tumor 1) et Bmp7 (bone morphogenetic protein 7), sont également indispensables à la survie de ces cellules. Un contrôle étroit est nécessaire pour assurer l'équilibre entre le maintien des progéniteurs - pour assurer la production de facteurs nécessaires à la ramification - et leur différenciation [3, 4]. La transition mésenchyme-épithélium est induite par la voie Wnt/ $\beta$-caténine, activée tout d'abord par Wnt9b (signal émanant du bourgeon urétéral), puis par Wnt4, exprimé dès le stade de vésicule rénale (Figure 1). L'activation de cette voie permet l'expression de gènes, dont ceux qui codent pour la cadhérine 6 (fetal kidney cadherin), le facteur de croissance Fgf8 (fibroblast growth factor 8), 